\title{
V. Beitrag zur Bluterkrankheit.
}

Von Dr. Theodor Klein in Offenbach a. M.

Trotz der ziemlich umfangreichen Litteratur iiber Bluterkrankheit halte ich es doch gerechtfertigt, einen Fall, wie den folgenden, den ich seit uıgefähr füıf Jahren zu beobachten Gelegenlieit hatte, zu veröffentlichen. In den meisten Abhandlungeı über Bluterkrankheit ist die Beschreibung der einzelnen Symptome, deren Bedentung und Behandlung im Verlältniss zu den theoretischieı Erörterungen über Aetiologie zu kurz gekommen. Dem Praktiker aber, der in der wenig erfreulichen Lage ist, einen Bluter oft Jahre lang behandeln zu müssen, dreht es sich vor allem $ı$ das „quid faciamus nos"? Wenn die folgende Krankengeschichte und einige daran geknüpfte Bemerkıngeı auch nur ein wenig zur Beantwortung der Frage beitragelı, so ist der Zweck dieser Veröffentlichung erfïllt.

K. B., Architekt, ist 24 Jahre alt. Sein Vater starb vor zwei Jahren an einem Herzleiden. Seime Mutter war einige Jahre geisteskrank und starb 40jährig an Apoplexie; sie sowohl wie ihre drei Sclıwestern sollen beim geringsteı Druck auf die Haut blaue Flecken gezeigt haben, sonst aber keinerlei Blıtere rscheiunngen. Der erste Sohn der einen Schwester war Blutes und starb im 14. Jahre nach einer Knieoperation. Der erste Sohn der zweiten Schwester verblutete sich beim Zahnziehen im 7. Lebensjahre. Die dritte Schwester blieb ledig. Grosseltern mütterlicherseits starben angeblich an Cholera im Jahre 1866. Weiteres ist von der Familie der Mutter nicht zu erfahren. Ein Bruder des K. B. lebt, ist 22 Jalıre alt und kein Bluter,

Er selbst sieht hochgradig anämisch aus, ist lang aufgeschossen und mager. Der Gesichtsausdruck ist der eines frühlzeitig gealterten Menschen. Organerkrankungen, insbesondere Volumenveränderung der Milz, sind nicht nachweisbar. Er hat als Kind die Masern und im 12. Jahre Diphtherie durchgemacht. Die Bluteranlage wurde angeblich bei der ersten Impfung entdeckt. Im 1. Jahre entstand durch Fall auf den Kopf eine kolossale Kopfgeschwulst, welche unter gleiclımässigem Druck durch eine dünne angepasste Kupferplatte und Eisumschläge allmïhlich zurückging. Im 6 . Jahre entstand aus einer kleinen Zungenlbisswunde eine starke, langandauernde Blutung, die endlich nach öfterem Brenuen nachliess. Im 10. Jahre Fall auf die Nase, in Folge dessen starke Blutung aus Nase und Muıd und unförmliches Unterlaufen des ganzen Gesichts. Im 14. Jahre trat eine starke Kniegeschwulst durch Stoss an eine Holzkante auf; schon das Sitzen auf einem Stuhl mit scharfer Kante genügte, um an der gedrückten Stelle des Oberschenkelss Schwellungen zu erzengen. Die oberflächlichen Blutungen unter die Haut waren bis zu dieser Zeit sehr häufig und stark. Viele Schmerzen aber hatten sie nicht verursacht, und die Schwellungen gingen bei Hochlagerung und Bettruhe nach einigen Tagen sclıon zurück. Mit dem 16. Lebensjahre wurden die Erscheinungen nach Angabe des B. wesentlich andere. Die sichtbaren Blutungen resp. Schwellungen wurden weniger, dafür aber traten heftige Schmerzen besonders in derı Gelenken auf, welche "rheumatischen ähılich" waren. Der geringste Druck oder Stoss verursachte tagelang Sclımerzen. Seit seinem 19. Jahre kenne iclı den B. persönlich, und zwar wurde ich damals wegen einer starken, nach einer Radfahrtour plötzlich aufgetretenen Knieschwellung, in Folge Gelenkblutung, zu Rathe gezogen. Gleichzeitig bestand eine Ellbogengelenkblutung.' Beide Schwellungen gingen nach einiger Zeit völlig zurück unter gleiclımässiger Compression. Kleine Verletzungen mit starken Blutverlusten kamen häufiger vor.

Ich verlor den Patienten ungefähr ein Jahr aus dem Auge, da derselbe von seinem Kassenarzte behandelt wurde. Da kam el cines Tages zu mir in die Sprechstunde, und ich fand in beiden Inguinalgegenden fast kindskopfgrosse, hart sich anfühlende Tumoren, die sich angeblich im Laufe der letzten Monate olne nachweisbare Ursachen entwickelt hatten. Das Gelıen war erschwert und schmerzhaft.

Nach der Anamnese - nicht nach Aussehen und Härte - stellte ich die Diagnose: "Blutergüsse". Er sollte nach seiner Angabe an eiıem der nächsten Tagre von einem auswärtigen Cliı urgen operirt werden, wollte mich zuvor jedoch 1 m meine Meinuıg fragen. Offenbar hatte, wenn die Sache sich wirklich so verhält, der messerfreudige Herr College keine Zeit gelıabt, die Anamınese genau aufzunehmen, sonst liätte die Diaghıose wahrscheinlich anders gelautet als "Chondrome des Femur". Er ging nun auf meinen Ratl einige Zeit nach Nauheim. Im Laufe der letzten zwei Jahre haben sich die Tumoren, besonders der linke, bedeutend verkleinert: der rechte ist noch deutliclı sicht- und fühlbar. Der Gang besserte sich jetzt bedeutend.

Vor ungefähr $1 \frac{1}{1} 2$ Jalıreı machte mir B. viele Sorgen. Er hatte eineı stark cariösen Backzahı, der ihm heftige Schnırzen verursachte; das überwuclıerte Zahnfleisch blutete sehr häufig. Eine Zeit lang war die Blutung durch Ausbrennen der Granulationen zu stillen, doch bald lialf auch das nicht wiehr, und das Blut sickerte fast immerzu aus dem 
umgebenden Zalınfleisch. An irgend einen Druckverband war nicht zu denken, da sich eine Periostitis mit hochgradiger Zahnfleischschwellung und Eiterbildung dazu gesellte. B. kam nach einigen Wochell, trotz Anwendung aller Mittel, durch Schmerzen und die fortwährenden kleinen Blutverluste so herunter, dass ich seiner dringenden Bitte, den Zalm ziehen zu lassen, nachgab, umsomehr, da er mir erklärte, er lasse sich sonst irgendwo den Zahn ziehen, ohne zu sagen, dass er Bluter sei; er halte das Leben so nicht mehr aus. Das wirkte, und ich bat einen hiesigen Zahnarzt, in meiner Gegenwart in dem hiesigen Schwesternhaus die Extraction vorzunehmen. Wir hatten alles fïr eine starke Blutung gerichtet und waren sehr erstaunt, dass es nach der Entfernung des Zahnes fast gar nicht blutete. Wir warteten noch eine halbe Stunde und gingen, angenehm enttäuscht, nach Hause. Nach drei Stunden aber wurde ich gerufen, da plötzlich eine starke Blutungr eintrat. Trotz aller angewandten Mittel - dauernder Compression mit eingedrückten Wattepfropfen, Eis, Hochlagerung des Kopfes, Ableitung auf Füsse und Darm, Ergotin und Styptica -- wollte sich dieselbe nicht stillen. Endlich nach zwei bangen Tagen hörte sie auf, wohl mehr in Folge der hochgradigen Blutleere, als der angewandten Mittel.

Es ist geradezu erstaunlich, was der Organismus in dieser Beziehung aushält; offenbar ist er bei Blutern noch ganz besonders auf grosse Blutverluste eingerichtet. In relativ kurzer Zeit erholte sich B. wieder.

Zwei Jahre lintereinander, im October 1897 und 1898 trat mehrere Tage lang Blut im Urin auf und klagte B. über Schmerzen in der Richtung des linken Ureter (wohl Niereublutung). Bei einigen Tagren Bettruhe und Diät verschwand das Blut wieder aus dem Urin.

Im Herbste vorigen und im Frühjalı dieses Jahres traten angeblich ohne besondere Ursachen starke schmerzhafte Scliwellungen an der Vorder- und Medianseite des linken Oberschenkels, in dessen oberem und mittlerem Drittel auf. Fluctuation war nicht zu fühlen. Die Schwellung ging nach Wochen zurück und hinterliess harte, zum Theil spitze, zum Theil scharfkantige, wie Exostosen sich ausnehmende, nicht verschiebliche Prominenzen.

Vor vier Wochen trat nach einer mehrstündigen Eisenbahnfahrt eine starke Schwellung am linken Oberschenkel auf, welche dessen mitt.leres Drittel ganz einnimmt und Fluctuation zeigt. Im Röntgenbilde sieht man deutlich neben dem Femur, aber meist durch hellere Stellen von ihın getrennt, also wohl hauptsächlich in der Muskelsubstanz liegend, die Sclıatten der eingedickten und verhärteten Blutmassen (Ossificationsprocess?), die Reste der Blutungen im Herbste 1898 und Frühjahr 1899.

Von den früheren ziemlich erheblichen Gelenkblutungen des linken Knies und des rechten Ellenbogens ist nichts mehr zu bemerken und blieb die Function der Gelenke vollständig erhalten. Es ist dies vielleicht für manchen, der Gelenkblutungen zum ersten Male in diesem Umfange sieht, in Bezug auf Prognose und Therapie nicht unwichtig.

Ausser ein bis zwei Woclien Bettrulıe wurde bei Kniegelenksblutungen ein feuchter und später trockener Compressionsverband angewendet. Die Frage, wie lange man einen Bluter bei Gelenkblutungen der unteren Extremitäten, insbesondere des Knies, strenge Bettruhe anempfehlen soll, möchte ich nach meiner Erfahrung dahin beantworten, dass man, wenn entzündliche Erscheinungen fehlen, schon nacli 8-14 Tagen mit gutsitzender Compressionsbinde oder Gelenkstrumpf vorsichtig das Aufstehen und Umhergehen versuchen lassen kann. Die Resorption geht dann jedenfalls rascher vor sich.

Da, wie wir gesehen haben, selbst die grossen Blutergüsse in die Muskelsubstanz, resp. unter die Haut - wenn auch sehr langsam, sich bedeutend verkleinem, ja fast ganz verschwinden können, so soll zu einem operativen Eingriff, selbst bei auf Monate hervorgerufener Functionsstörung der Extremitäten wegen der damit verbundenen grossen Lebensgefahr bei Blutern nicht geschritten werden, es sei denn, dass Verjauchung der Masse dies unbedingt nöthig macht. Jedenfalls aber soll man selbst bei harten Geschwülsten, welche als Chondrome, Osteome u. s. w. imponiren können, immer bei der Differentialdiagnose an die Möglichkeit einer Blutgescliwnlst denken, um unangenelıne Folgen zu vermeiden, wie sie schon öfter vorgekommen sein sollen.

Verantwortungsvoll und nur im Einzelfalle zu beantworten ist die Frage, ob man bei einem Bluter eine Zahnextraction vornelomen soll. Mit Recht wird immer davor gewarnt; in der Praxis giebt es aber Fälle, wo man sich gezwungen sieht, ,ja“ zu sagen, wie in dem vorliegenden. Die fortwährenden Blutungen, Schmerzen, Schlaflosigkeit, Unvermögen zu essen bei der hochgradigen Periostitis hatten den B. bis aufs äusserste heruntergebracht, sodass ohne Extraction ein schlimmer Ausgang zu befürchten war. Diese Ueberlegung und die verzweifelte Drohung des armen Teufels bestimmten mich, der Extraction zuzustimmen, jedoch erst, nachdem ich ihm und seinen Verwandten die Gefahr derselben nochmals deutlich klargelegt hatte.

Dass der Ausgang ein glïcklicher war; trotz zweitägiger starker Blutung, haben wir gesehen; wenn auch unsere Mittel (vor allem die Compression) die Blutung nicht direkt gestillt haben, jedenfalls haben sie den Zweck erfüllt, den Abfluss des Blutes zu verlangsamen und die Blutmengenverminderung im Kreislauf nicht zu schnell eintreten zu lassen, und das ist sehr wichtig. Aber ferner liaben wir dabei erfalıren, dass auch bei Blutern niclit immer die Blutıng sofort nach dem Eingriffe oder der Verletzung eintritt. Man muss deshalb die nächsten Stunden und Tage den Patienten im Auge behalten, da jede Minute die Blutung kommen, resp. wiederkommen kann, besonders im Mund durch Zungen- oder Kaubewegung.

Erwähnt sei auch, dass in letzter Zeit von Zahnärzten bei starken Blutungen "Stypticin" mit Erfolg angewendet worden sein soll. Ich habe keine Erfahrung darüber. Zweckmässig wird es auch sein, die ersten Tage nach der Extraction auf die Ernährung per Os möglichst zu verzichten und ernährende Klysmen zu geben.

Wie von den meisten Autoren angegeben, finden wir auch in unserm Falle, dass im Frühjahre und Herbste die Hauptblutungen stattfinden, es wäre demnach besonders $11 \mathrm{~m}$ diese Zeiten den Blutern Vorsicht anzuempfehlen und unnötlige Reisen und Anstrengungen zu verbieten. Für sehr wesentlich in therapeutischer und prophylaktischer Hinsicht halte ich eine möglichste Einschränkung der Flüssigkeitszufuhr als Entlastung der Kreislaufsorgane, und wäre besonders in der Blutersaison (Herbst und Frühjahr) eine streng durchgeführte Trockendiät am Platze. Das oft als Panacee angepriesene Glaubersalz wirkt jedenfalls auch nur durch Fliissigkeitsentziehung in den Darm. Selbstverständlich ist eine Vermeidung erregender Stoffe, wie Alkohol, Kaffee u. s. w. geboten.

Von lokalen Blutstillungsmitteln, z. B. bei Verletzungen, gebührt meiner Erfahrung nach der Dauercompression der Vorlang. Alle Mittel, welche eine Schorfbildung bezwecken, sind deshalb weniger am Platze, weil es nach Abstossung derselben leiclit wieder blutet und ausserdem, wie bei Eisenchloridanwendung die Uebersiclit der blutenden Stelle erschwert wird.

Die vielen andern mehr oder ininder wirksamen Mittel übergehe ich hier: nur sei noch erwähnt, dass ein College') in einem Falle bei einer stark blutenden Schläferıwunde, naclı fruchtloser Anwendung der verschiedensten Mittel, eine rasche Blutstillung, resp. Gerinnung dadurch erzielte, dass er Blut eines gesunden Menschen in die Wunde einspritzte.

F. Krause behandelte kürzlich, ermuthigt durch die günstige Einwirkung bei Aortenaneurysma, einen 15jährigen Bluter durch Einspritzung einer $1-2 \frac{1}{2} \%$ igen Gelatinekochsalzlösung, theils subcutan, tlıeils in das blutende Gelenk selbst und war mit dem Erfolge sehr zufrieden. (Münchener medicinische Wochenschrift No 47, S. 1578. $)^{3}$ )

Hiermit will ich diese kleine Abliandlung beschliessen, vielleicht kann ich in späteren Jahren über das fernere Schicksal unseres Bluters berichten. 